\title{
Comparaison des peuplements chironomidiens du lac de l'Abbaye obtenus par différentes méthodes d'échantillonnage. Intérêts de la récolte des exuvies nymphales Comparison of the chironomid communities of Lake Abbaye (Jura, France) collected using five different sampling methods. Avantages of the pupal exuviae sampling
}

\author{
V. Verneaux et L. Aleya
}

Volume 12, numéro 1, 1999

URI : https://id.erudit.org/iderudit/705343ar

DOI : https://doi.org/10.7202/705343ar

\section{Aller au sommaire du numéro}

\section{Éditeur(s)}

Université du Québec - INRS-Eau, Terre et Environnement (INRS-ETE)

\section{ISSN}

0992-7158 (imprimé)

1718-8598 (numérique)

\section{Découvrir la revue}

Citer cet article

Verneaux, V. \& Aleya, L. (1999). Comparaison des peuplements chironomidiens du lac de l'Abbaye obtenus par différentes méthodes d'échantillonnage. Intérêts de la récolte des exuvies nymphales. Revue des sciences de l'eau / Journal of Water Science, 12(1), 45-63. https://doi.org/10.7202/705343ar

\section{Résumé de l'article}

Une étude des espèces chironomidiennes du lac de l'Abbaye a été effectuée au cours de l'année 1993. Le répertoire spécifique a été établi à partir de l'étude des peuplements imaginaux, nymphals et larvaires échantillonnés selon 5 méthodes: récolte des imagos au filet entomologique, récolte des exuvies nymphales, mise en élevage de stades pré-imaginaux, prélèvements de larves dans les sédiments et mise en place de substrats artificiels. Le peuplement chironomidien obtenu est constitué de 69 espèces. Une comparaison de la composition des peuplements obtenus par les différents modes d'échantillonnage permet de mettre en évidence les particularités de chaque méthode. La récolte des exuvies nymphales semble être la méthode la plus appropriée pour l'établissement d'un répertoire spécifique. Deux espèces dominantes du lac Chironomus anthracinus et Psilotanypus rufovittatus témoignent du caractère polyhumique et désoxygéné du lac. Cependant, la présence simultanée en forte proportion de Tanytarsus niger, Cladotanytarsus iucundus et $\mathrm{C}$. atridorsum témoignent de la faible production pélagique et de la température froide du lac susceptible de minimiser les effets de la désoxygénation sur la communauté chironomidienne. 


\title{
Comparaison des peuplements chironomidiens du lac de l'Abbaye obtenus par différentes méthodes d'échantillonnage. Intérêts de la récolte des exuvies nymphales
}

\author{
Comparison of the chironomid communities \\ of Lake Abbaye (Jura, France) collected using \\ five different sampling methods. \\ Avantages of the pupal exuviae sampling.
}

\section{VERNEAUX* et L. ALEYA}

Reçu le 17 novembre 1997, accepté le 5 mai 1998**.

\section{SUMMARY}

A study of chironomid species was undertaken in Lake Abbaye during the year 1993. The list of the species obtained resulted from the use of 5 different sampling methods: (i) adults caught with an entomological net; (ii) pupal exuviae sampling at the sites where they were accumulated; (iii) rearing of larvae; (iv) sediment sampling and (v) sampling of larvae using artificial substrates. The chironomid community of Lake Abbaye consisted of 69 species. A comparison among the different chironomid communities stresses the particularity of each sampling method. This study clearly showed that pupal exuviae sampling was the more appropriate method to establish a list of species. The chironomid community of Lake Abbaye is dominated by two species (Chironomus anthracinus, Psilotanypus rufovittatus) that reflect the polyhumic and hypoxic characteristics of the lake. Simultaneously, the great proportion of three other species (Tanytarsus niger, Cladotanytarsus iucundus, $C$. atridorsum) indicates that the lake has low phytoplanktonic biomass and low temperature that probably minimize the impact of hypoxic conditions on the chironomid community.

A study of chironomid species was undertaken in Lake Abbaye during the year 1993. Lake Abbaye is located $910 \mathrm{~m}$ a.s.l., in a karstic area in the east of France near Switzerland (Figure 1 ). It covers 80 ha with a maximum depth of $19 \mathrm{~m}$ and a mean depth of $7.2 \mathrm{~m}$. Profundal sediment has a high level of organic matter (30.2\% dry weight) of mainly terrestrial origin. The accumulation of the organic matter resulted in hypoxic conditions at depth during the summer stratification. The lake is rather cold (maximum surface temperature: $23^{\circ} \mathrm{C}$ ), holodimictic, and is ice-covered from late November to early April.

The list of chironomid species obtained resulted from the use of 5 different sampling methods: (i) adults caught with an entomological net; (ii) pupal exu- 
viae sampling at the sites where they were accumulated; (iii) rearing of larvae; (iv) sediment sampling and (v) sampling of larvae using artificial substrates. The sampling site locations are indicated in Figure 1. The present paper gives results and discussions about i) the taxonomic richness and composition of the total chironomid community, ii) the composition of the different communities collected and the characteristics of the five sampling methods, iii) a characterization of Lake Abbaye according to the dominating species.

\section{Total chironomid community}

The chironomid community of Lake Abbaye consisted of 69 species (Table 1). Compared to other European lakes, this community showed a rather high richness. In some great lakes, however, the number of chironomid species amounted to 140 or 168 species (Lake Innaren, Lake Constance). These particularly high richnesses probably resulted from the great number of substrata according to the great surface of the littoral zone of these lakes. The chironomid community of Lake Abbaye is specific to a mountain area as shown by the high proportion of species collected (82\% of the total richness) found by SERRA-TOSIO and LAVILLE (1991) at altitudes exceeding $500 \mathrm{~m}$. According to REISS and FITTKAU (1971), the presence of two particular species (Tanytarsus niger and $T$. miriforceps) reflects the glacial origin of the lake.

\section{Composition of the different chironomid communities}

Sampling the pupal exuviae allowed us to collect more than $90 \%$ of the species with less than 5000 individuals whereas by catching adults this percentage did not exceed $79 \%$ with more than 28000 individuals collected and identified. By rearing larvae both the number of species ( 46 species, $66 \%$ of the total richness) and the number of emerging adults (1 386 individuals) were low. The least efficient method appeared to be the sediment sampling, which allowed the identification of only 19 taxa (genera and species) compared to the 31 taxa collected by artificial substrates. This study clearly showed that pupal exuviae sampling was the most appropriate method to establish a list of species yielding the greatest ratio between species richness and the effectives.

A comparison of the major contributed species among the different communities led to the conclusion that the sampling method strongly affects community composition. Whereas in adult population (Figure 2) the dominating species (relative density $=10 \%$ ) were Tanytarsus niger, Ablabesmyia monilis, A. longistyla and Polypedilum nubeculosum, the community of pupal exuviae (Figure 2) was dominated by Cladotanytarsus iucundus and that obtained by larvae rearing (Figure 2) showed two other (genera and species) dominating species (Psilotanypus rufovittatus and Einfeldia dissidens).

The study of the chironomid community by catching adults presented two major difficulties. The first resulted from the sampling of adults that did not emerge from the lake itself. The second problem involved a better sampling of the bigger and/or the more accessible swarms. This method led to an overestimate of the species richness of the lake and modified the species proportions in the community. For example, the great proportion of two species, Ablabesmyia monilis and $A$. longistyla, in the adult community was observed in none of the other communities (Figure 2).

In both larval populations (Figure 3), the genera Chironomus, Tanytarsus, Cladotanytarsus and Procladius dominated. The use of artificial substrates induced, however, a lot of modifications in some genera contributions. For example, the genus Einfeldia was collected at higher relative density in artificial substrates than in the sediment samples. Moreover, a lot of taxa (Glyptotendipes, Microtendipes, Paratendipes, Polypedilum, Cricotopus, Parakiefferiella) were exclusively collected, in larval communities, with the artificial substrates. Despite the well-known differences between the benthic communities collected insitu and those sampled by artificial substrates (ROSENISERG et RESH, 1982), we 
think such a technique allows a correct study of the taxonomic richness. The use of identical substrates in different lakes should allow one to define a potential community also related solely to the water quality.

\section{Characterization of the Lake Abbaye}

The study of the taxonomic composition of five different chirononid communities stresses the difficulty in characterizing the lake according to the dominating species. However, comparison between larval community (Figure 3) and imagos or exuviae populations (Figure 2) led to the conclusion that Lake Abbaye could be classified in the category of lakes with Tanytarsus (specially T. niger) and Cladotanytarsus ( $C$. iucundus and $C$. atridorsum). The particularity of Lake Abbaye is that two groups of species dominated the community. The first, constituted by the aforementioned three species, is dominating in oligotrophic lakes (BRUNDIN, 1949). The second group, represented by Chironomus anthracinus and Psilotanypus rufovittatus, reflected the polyhumic and hypoxic characteristics of the lake. These results indicate that Lake Abbaye has low phytoplanktonic biomass but high organic matter content in its sediment, which originates from terrestrial or littoral inputs (VERNEAUX et al., 1987). The quite low temperature of the water probably minimizes both the degradation of the organic matter and the impact of hypoxic conditions on the chironomid community.

\section{Conclusion}

The present paper clearly demonstrates the importance of the sampling method used to study chironomid communities. Sampling the pupal exuviae in the accumulation zones is the most appropriate method to study chironomids. In order to contribute to a better understanding of the relations between the chironomid species and the environmental conditions, our further research will concentrate on a study of the bathymetric distribution of these species. For this purpose, pupal exuviae will be sampled at different isobaths simultaneously with chemical and physical analyses of the water and sediments. The drift phenomenon of exuviae that could affect the bathymetric pattern of species seemed to be avoided when a criterion of minimum occurrence was taken into account (VERNEAUX, 1996).

Key-words: lakes, chironomidae, pupal exuviae.

Une étude des espèces chironomidiennes du lac de l'Abbaye a été effectuée au cours de l'année 1993. Le répertoire spécifique a été établi à partir de l'étude des peuplements imaginaux, nymphals et larvaires échantillonnés selon 5 méthodes : récolte des imagos au filet entomologique, récolte des exuvies nymphales, mise en élevage de stades pré-imaginaux, prélèvements de larves dans les sédiments et mise en place de substrats artificiels. Le peuplement chironomidien obtenu est constitué de 69 espèces. Une comparaison de la composition des peuplements obtenus par les différents modes d'échantillonnage permet de mettre en évidence les particularités de chaque méthode. La récolte des exuvies nymphales semble être la méthode la plus appropriée pour l'établissement d'un répertoire spécifique. Deux espèces dominantes du lac Chironomus anthracinus et Psilotanypus rufovittatus témoignent du caractère polyhumique et désoxygéné du lac. Cependant, la présence simultanée en forte proportion de Tanytarsus niger, Cladotanytarsus iucundus et $C$. atridorsum témoignent de la faible production pélagique et de la température froide du lac susceptible de minimiser les effets de la désoxygénation sur la communauté chironomidienne. 


\section{1 - INTRODUCTION}

L'étude de la macrofaune benthique a fait l'objet de nombreux travaux dans les milieux d'eau douce (MACAN, 1961; HYNES, 1970 ; MACAN, 1970; BRINKHURST, 1974). Au sein des écosystèmes lacustres cette macrofaune est essentiellement dominée par les Diptères Chironomidae qui représentent plus de $50 \%$ des effectifs dénombrés (BRUNDIN, 1949 ; OLIVER, 1960 ; GRIMAS, 1961; SLACK, 1965 ; BONOMI et RUGGIU, 1966 ; WIEDERHOLM et ERIKSSON, 1977 ; AAGAARD, 1978 ; LINDEGAARD, 1979). Cette importance quantitative majeure des Diptères Chironomidae semble être étroitement liée à la stratégie de survie qu'ils adoptent en périodes de contraintes mésologiques (température, oxygène, $\mathrm{pH}$...). À ce sujet, CRANSTON (1995) rapporte que certaines espèces peuvent se développer dans des milieux considérés comme très hostiles aux autres composantes de la faune entomologique. Par ailleurs, l'étude des Diptères chironomidés contribue à la compréhension du fonctionnement des écosystèmes lacustres en raison notamment de la capacité de ces organismes à pouvoir intégrer la variabilité des phénomènes physiques et chimiques observée tant au niveau de la masse d'eau que du sédiment (VERNEAUX et ALEYA, 1997a). De plus, de par leur contribution au recyclage des biomasses bactérienne et protozooplanctonique, les Diptères chironomidés participent activement à l'optimisation du fonctionnement de la boucle microbienne (FENCHEL, 1987 ; PORTER et al., 1985 ; SHERR and SHERR, 1984 ; SHERR et al., 1986).

Les études réalisées sur la faune chironomidienne lacustre témoignent d'une grande variabilité des méthodes d'échantillonnage dépendant du stade du cycle biologique étudié (larves, imagos ou exuvies nymphales). Dans la grande majorité des études seuls les stades larvaires sont récoltés et leur prélèvement dans les sédiments avec une benne de type Ekman est le plus souvent cité dans la littérature (JOHNSON et WIEDERHOLM, 1989 ; RIERADEVALL et PRAT, 1991 ; VEIJOLA, 1996 ; DINSMORE et PREPAS, 1997). Certains auteurs complètent l'échantillonnage des sédiments par un échantillonnage de substrats variés, en zone littorale, par l'utilisation de filets de type filet de Zürber (JOHNSON et al., 1993 ; BAZZANTI et al., 1997). Les substrats artificiels sont assez rarement utilisés excepté pour pallier aux difficultés d'échantillonnage de certaines zones littorales et sublittorales essentiellement rocheuses (LAVILLE, 1974) ou pour effectuer des études de dynamique de colonisation par le macrobenthos (CASEY et KENDALL, 1996). Quelque soit le matériel et la méthode choisie pour la récolte des stades larvaires, leur détermination est essentiellement générique. L'obtention de données spécifiques nécessite la récolte des imagos, des exuvies nymphales ou la mise en élevage de stades préimaginaux (larves ou nymphes) jusqu'à obtention des imagos et des exuvies nymphales associées. Les études récentes des espèces chironomidiennes lacustres, assez peu nombreuses, sont pour la plupart basées sur un échantillonnage des imagos par l'utilisation de pièges à émergence (LAVILLE, 1971; SHERK et RAU, 1996) ou de filet entomologique (HIRABAYASHI et HAYASHI, 1996). Alors que la récolte des exuvies nymphales est à l'origine de nombreuses études des peuplements chironomidiens des eaux courantes (COFFMAN, 1973; WILSON et BRIGHT, 1973 ; FRANQUET et PONT, 1996 ; BAZERQUE et al., 1989 ; EVRARD, 1994 ; FEND et CARTER, 1995) cette méthode est peu citée pour l'étude des peuplements lacustres (HUMPHRIES, 1938 ; BUSKENS, 1989; CASAS et VILCHEZQUERO, 1996). La grande diversité des méthodes d'étude des peuplements chiro- 
nomidiens rend problématique la comparaison des résultats obtenus dans différents lacs tant au niveau de la variété que de la composition des peuplements. Or, très peu d'étude proposent une analyse simultanée et dans un écosystème unique des différents types de peuplements chironomidiens afin d'établir les particularités de chacun et de comparer les méthodes d'échantillonnage (RUSE, 1995).

De plus, à notre connaissance, les études de ce compartiment faunistique réalisées à un niveau de détermination spécifique sont rares. En conséquence, nous nous sommes proposés dans le présent travail d'étudier les trois types de peuplements chironomidiens (larvaire, nymphal et imaginal) du lac de l'Abbaye en insistant sur la détermination spécifique des organismes récoltés qui ont été obtenus par l'application de cinq méthodes d'échantillonnage.

\section{2 - MATÉRIELS ET MÉTHODES}

\subsection{Site d'étude}

Le lac de l'Abbaye est situé à $910 \mathrm{~m}$ d'altitude, dans la partie septentrionale du massif Jurassien, à l'est de la France (figure 1). Ce massif calcaire, plissé lors de la formation des Alpes, a été fortement remodelé au cours de la période de glaciation du Würm. Le lac de l'Abbaye doit sa formation au surcreusement glaciaire d'une dépression synclinale secondairement imperméabilisée par des dépôts morainiques. Constitué d'une cuvette isolée assez peu profonde (profondeur max. : $19 \mathrm{~m}$, profondeur moy. : 7,2 m), sans vecteur fluviatile majeur, ce lac de petites dimensions (surface : 80 ha, longueur max. : $2125 \mathrm{~m}$, largeur max.: $600 \mathrm{~m}$ ) est caractérisé par une accumulation de matière organique (teneur moyenne à la profondeur maximale : $30,16 \%$ matière sèche) dont l'origine est essentiellement allochtone (tourbières et forêts environnantes) (VERNEAUX et al., 1987). Ce comblement organique se traduit par une période assez longue de désoxygénation des eaux profondes (durée de l'anoxie à la profondeur maximale : 3 mois) qui survient en période de stratification thermique et chimique (VERNEAUX et ALEYA, 1997b). La réoxygénation des eaux est assurée par les périodes de brassage (printanière et automnale) et par des émergences karstiques profondes.

\section{2 Échantillonnage}

Le répertoire spécifique du peuplement chironomidien du lac de l'Abbaye a été établi à partir de l'échantillonnage des différents stades de développement de la faune chironomidienne (imagos, exuvies nymphales et larves). Les prélèvements ont été effectués au cours de l'année 1993.

\subsubsection{Imagos}

Les imagos ont été récoltés au filet entomologique, toutes les semaines, du 7/04/93 (i.e. avant le brassage des eaux) au 19/10/93 et sous conditions météorologiques favorables (vents faibles à nuls, absence de fortes précipitations). À chaque campagne, la durée de récolte des imagos a été répartie, en soirée, en trois pério- 


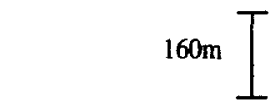

Echantillonnage des exuvies
Echantillonnage des imagos

- Substrats artificiels

+ Echantillonnage des sédiments
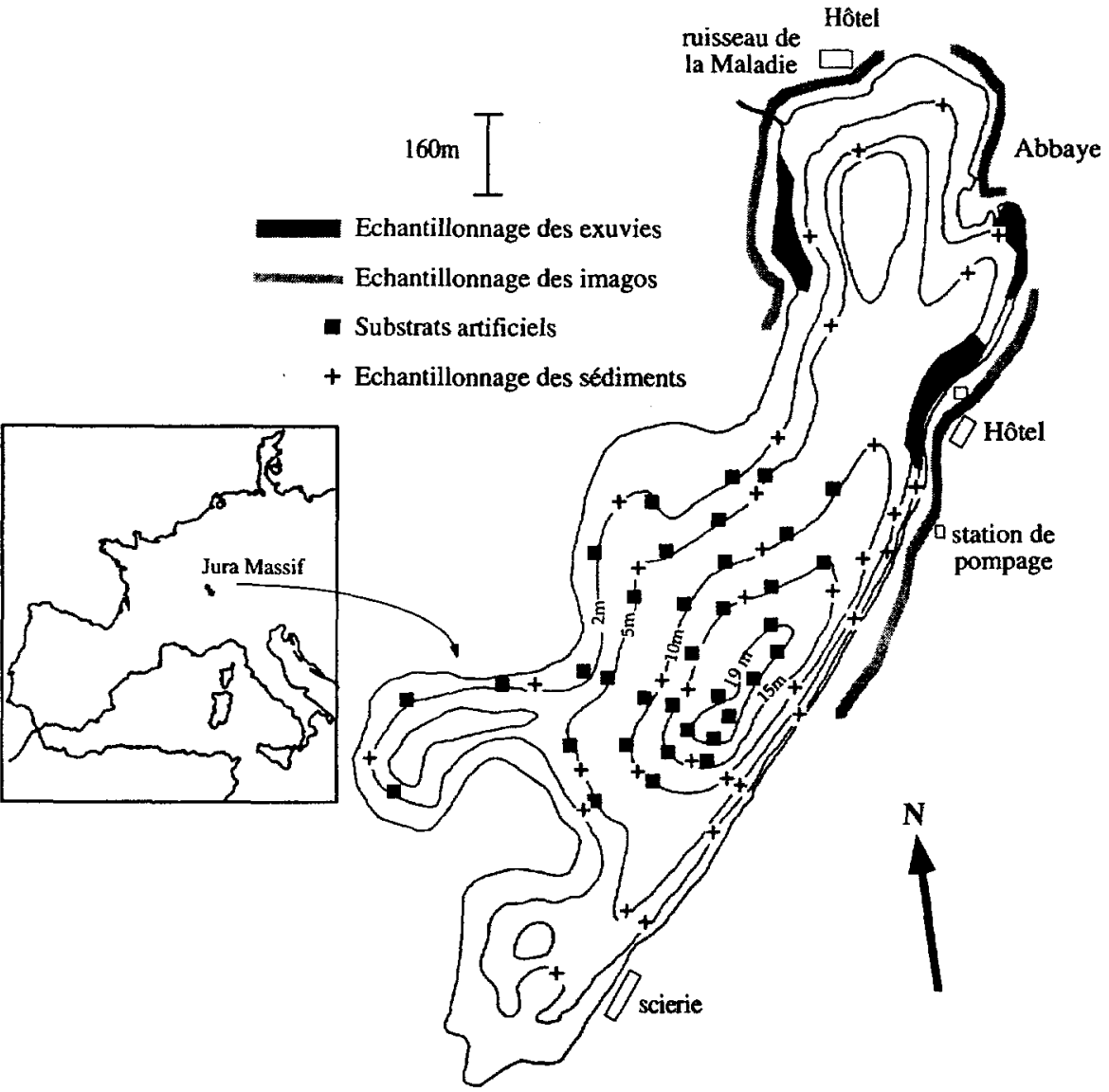

$\mathbf{N}$

Hôtel

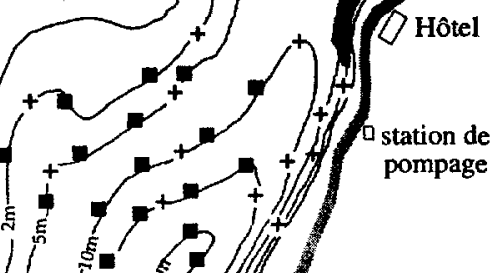

Figure 1 Localisation géographique, carte bathymétrique du lac de l'Abbaye et emplacement des sites d'échantillonnage des peuplements imaginaux, nymphals et larvaires.

Bathymetric map of Lake Abbaye, location of the sampling sites for adults, pupal exuviae and larvae.

des d'une heure sur les trois sites jalonnant le pourtour le plus accessible du lac (figure 1). Les imagos formant généralement des essaims mono-spécifiques (LINDEBERG, 1964), le filet entomologique a été équipé à son extrémité inférieure d'un pas de vis sur lequel s'adaptent des piluliers d'une contenance de $40 \mathrm{~mL}$. Ce dispositif présente l'avantage de minimiser le temps de tri de l'ensemble des individus récoltés. Les imagos sont conservés dans de l'eau additionnée de formol $10 \%$. Au laboratoire, ils sont montés entre lame et lamelle selon la méthode décrite par PINDER (1978) après avoir subi un traitement de 12 heures, à froid, dans une solution de $\mathrm{KOH} 10 \%$. Seuls les imagos mâles ont été déterminés et comptés.

\subsubsection{Exuvies nymphales}

La récolte des exuvies nymphales s'est déroulée simultanément à celle des imagos. L'échantillonnage a été effectué à la surface de l'eau, dans les zones 
d'accumulation (figure 1) et à l'aide d'un filet à ouverture rectangulaire de $125 \mu \mathrm{m}$ de vide de maille. La détermination des espèces a été réalisée d'après l'ouvrage de LANGTON (1991).

\subsubsection{Larves et élevage des stades pré-imaginaux}

Le peuplement larvaire a été obtenu par relevage mensuel (de mai à novembre) d'un total de 126 substrats artificiels immergés, en avril 1993, à différentes profondeurs $(-2 \mathrm{~m},-5 \mathrm{~m},-10 \mathrm{~m},-15 \mathrm{~m},-19 \mathrm{~m})$. Les substrats utilisés résultent d'une simplification du prototype élaboré pour la faune des milieux lotiques (MALÉSIEUX, 1976) et expérimenté en lac en 1991 (GIMARET, 1995). Ces substrats sont constitués d'un disque en béton dans lequel sont intégrés différents matériaux de granulométrie et de nature variables (graviers, sables, brosses synthétiques, anfractuosités). L'ensemble du dispositif (surface colonisable: $1 / 40 \mathrm{~m}^{2}$ ) est entouré d'un filet cylindrique (vide de maille : $250 \mu \mathrm{m}$ ) qui empêche la fuite des organismes lors du relevage des substrats artificiels (ROSENBERG et RESH, 1982). En phase de colonisation ce filet est replié à la base des substrats autorisant une colonisation par une faune de toute taille.

Chaque mois, 18 substrats artificiels sont relevés. Neuf d'entre eux sont destinés à la détermination générique et/ou spécifique des larves, les neuf autres sont utilisés pour la mise en élevage des stades préimaginaux jusqu'à obtention des imagos et des exuvies nymphales associées. Pour la mise en élevage, les substrats artificiels ainsi que les sédiments accumulés dans le filet de relevage sont placés, sans tri préalable, dans des cuves en matière plastique transparente (diamètre : $24 \mathrm{~cm}$, hauteur : $15 \mathrm{~cm}$ ). Seuls les principaux prédateurs (Sialidae, Odonates) sont éliminés. Les cuves sont ensuite remplies d'eau du lac jusqu'à une hauteur permettant le recouvrement des substrats puis transportées dans une glacière afin de limiter la mortalité des larves (SERRA-TOSIO, 1971). Au laboratoire, elles sont placées à proximité d'une source lumineuse et équipées d'un diffuseur d'air comprimé.

Afin de compléter l'inventaire des taxons larvaires récoltés sur substrats artificiels, des prélèvements de sédiment ont été effectués à l'aide d'une benne Ekman, au mois d'avril 1993. Les profondeurs prospectées sont identiques aux profondeurs d'immersion des substrats artificiels et le nombre d'échantillons de sédiment prélevé sur chaque isobathe est proportionnel à leur longueur (VERNEAUX et al., 1993). Les sites de mise en place des substrats artificiels et de prélèvements de sédiments sont présentés sur la figure 1.

\section{3 - RÉSULTATS}

\subsection{Variété taxinomique}

Les différentes méthodes d'échantillonnage utilisées pour l'étude du peuplement chironomidien du lac de l'Abbaye ont permis de recenser 69 espèces dont les effectifs relatifs sont présentés dans le tableau 1.

Près de $95 \%$ des espèces (65 espèces) ont été obtenus par l'échantillonnage des exuvies nymphales, dans les zones d'accumulation, pour un effectif de seule- 
Tableau 1 Répertoire spécifique du peuplement chironomidien récolté dans le lac de l'Abbaye et effectifs relatifs de chaque taxon dans les différents peuplements échantillonnés. S.A. : substrats artificiels. pr : présence (effectif relatif $<0,01 \%$ ).

Table 1 List of the chironomid species collected in Lake Abbaye and relative number of individuals for each taxon in the different sampled communities. S.A.: artificial substrates. pr: presence (relative density $<0.01 \%$ ).

\begin{tabular}{|c|c|c|c|c|c|c|}
\hline Genres & Espèces & $\begin{array}{l}\text { Larves } \\
\text { S.A. }\end{array}$ & $\begin{array}{c}\text { Larves } \\
\text { sédiment }\end{array}$ & Imagos & Exuvies & Élevages \\
\hline Chironomini & & 50,92 & 22,47 & 20,02 & 28,5 & 36,12 \\
\hline \multirow[t]{5}{*}{ Chironomus } & & 15,09 & 14,60 & 0,16 & 8,86 & 0,86 \\
\hline & anthracinus (Zett) & & & 0,15 & 8,57 & 0,58 \\
\hline & nuditarsis (Keyl) & & & & 0,02 & 0,14 \\
\hline & plumosus (L.). & & & pr & 0,21 & 0,07 \\
\hline & pseudothummi (S.) & & & pr & 0,06 & 0,07 \\
\hline \multirow{4}{*}{$\begin{array}{l}\text { Cladopelma } \\
\text { Cryptochironomus }\end{array}$} & viridula (L.) & 2,42 & 0,11 & 0,57 & 2,67 & 3,46 \\
\hline & & 0,08 & 0,27 & pr & 0,08 & \\
\hline & psittacinus (Mg.) & & & pr & 0,02 & \\
\hline & supplicans (Mg.) & & & & 0,06 & \\
\hline \multirow[t]{3}{*}{ Dicrotendipes } & & 1,46 & 3,18 & 0,18 & 1,44 & 3,47 \\
\hline & modestus (Say) & 1,37 & 2,86 & 0,03 & 1,13 & 2,89 \\
\hline & nervosus (Staeg.) & 0,08 & 0,32 & 0,15 & 0,31 & 0,58 \\
\hline \multirow[t]{3}{*}{ Einfeldia } & & 20,09 & 1,54 & 0,06 & 4,02 & 15,29 \\
\hline & carbonaria (Mg.) & & & 0,05 & 2,13 & 3,39 \\
\hline & dissidens (Walk.) & & & 0,01 & 1,90 & 11,90 \\
\hline \multirow[t]{3}{*}{ Endochironomus } & & 7,15 & 0,11 & 0,11 & 0,86 & 5,85 \\
\hline & albipennis (Mg.) & 6,98 & 0,11 & 0,02 & 0,67 & 3,90 \\
\hline & tendens (Fabr.) & 0,17 & & 0,09 & 0,19 & 1,95 \\
\hline Glyptotendipes & gripekoveni (K.) & 0,27 & & 0,09 & & \\
\hline Microtendipes & chloris (Mg.) & 1,72 & & 0,07 & 7,84 & 3,17 \\
\hline Pagastiella & orophila (Edw.) & 0,09 & 0,96 & 0,01 & 0,06 & \\
\hline \multirow[t]{7}{*}{ Parachironomus } & & 0,56 & 1,43 & 0,20 & 0,13 & 0,86 \\
\hline & arcuatus (Goetg.) & & & pr & 0,04 & 0,43 \\
\hline & biannulatus (Staeg.) & & & 0,03 & 0,04 & 0,07 \\
\hline & tenuicaudatus (Mal.) & & & 0,01 & 0,02 & 0,22 \\
\hline & varus gr. (Goetg.) & & & 0,15 & & 0,07 \\
\hline & vitiosus gr. (Goetg.) & & & 0,02 & & 0,07 \\
\hline & Pe4 (Langton) & & & & 0,02 & \\
\hline \multirow[t]{3}{*}{ Paratendipes } & & 0,12 & & $\theta, 12$ & 1,00 & 0,50 \\
\hline & albimanus (Mg.) & 0,10 & & 0,01 & 1,00 & 0,50 \\
\hline & nudisquama (Edw.) & 0,02 & & 0,11 & & \\
\hline Pentapedilum & sordens (V.D.W.) & 0,10 & & 2,90 & & 0,07 \\
\hline Phaenopsectra & flavipes (Mg.) & 0,02 & & 0,01 & 0,04 & \\
\hline Polypedilum & nubeculosum (Mg.) & 1,67 & & 14,94 & 1,42 & 2,16 \\
\hline Tribelos & intextus (Walk.) & & 0,27 & & 0,04 & \\
\hline Tripodura & $\mathrm{sp}$ & 0,08 & & 0,59 & 0,04 & \\
\hline Pseudochironomini & & 0,72 & 0,16 & 2,40 & 0,58 & 0,22 \\
\hline Pseudochironomus & prasinatus (Staeg.) & 0,72 & 0,16 & 2,40 & 0,58 & 0,22 \\
\hline Tanytarsini & & 31,17 & 55,46 & 45,27 & 46,64 & 34,10 \\
\hline Cladotanytarsus & & 14,48 & 27,25 & 18,74 & 22,10 & 14,28 \\
\hline & atridorsum (Kief.) & & & 7,98 & 8,92 & 7,86 \\
\hline & iucundus (Hirv.) & & & 6,95 & 10,78 & 1,37 \\
\hline & lepidocalcar (Krūg.) & & & & 0,02 & \\
\hline & nigrovittatus (Goet.) & & & & 2,04 & 4,04 \\
\hline & pallidus (Kief.) & & & 3,81 & 0,24 & 1,01 \\
\hline Paratanytarsus & tenuis (Mg.) & 1,74 & 2,08 & 0,03 & 1,25 & 0,65 \\
\hline
\end{tabular}


Tableau 1 (Suite)

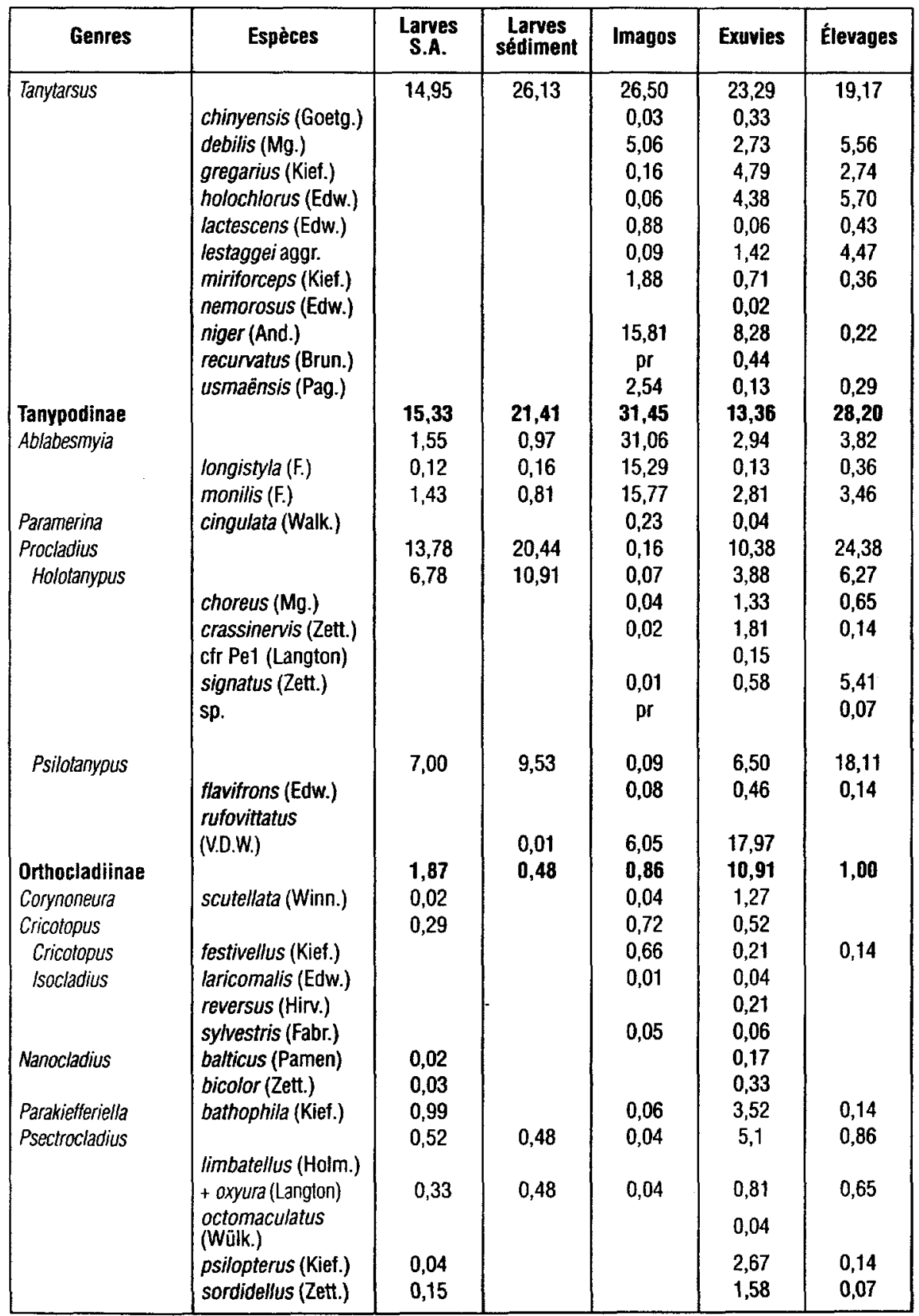

ment 4797 individus. La récolte des imagos au filet entomologique n'apporte en revanche que 55 espèces alors que 28193 individus mâles ont été déterminés. Seulement 48 espèces et 1386 individus ont émergé à la suite de la mise en élevage des stades pré-imaginaux. 
La variété taxonomique la plus faible (19 taxons) est obtenue par les prélèvement des sédiments avec un effectif également faible de 1879 individus alors que le peuplement larvaire récolté sur substrats artificiels présente une variété de 31 taxons et un effectif de 28193 larves. Si, à un niveau de détermination identique (genre et espèce), la récolte des imagos, des exuvies nymphales et des larves sur substrats artificiels ont permis de recenser un nombre identique de taxons ( 31 taxons), la proportion la plus élevée entre le nombre de taxons récoltés et les effectifs est assurée par l'échantillonnage des exuvies nymphales.

\subsection{Composition des peuplements chironomidiens}

Le peuplement global est dominé par les Chironomini et les Tanytarsini qui représentent respectivement $42,6 \%$ et $25,7 \%$ de la variété spécifique. Les Orthocladiinae et les Tanypodinae participent de manière similaire à la variété du peuplement (respectivement 17 et $15 \%$ de la variété totale) et le genre Tanytarsus présente le plus grand nombre d'espèces (11 espèces soit près de $16 \%$ de la variété totale).

Le peuplement imaginal (figure 2) est largement dominé par les espèces Tanytarsus niger, Ablabesmyia monilis, A. longistyla et Polypedilum nubeculosum. Ces espèces représentent à elles seules plus de $60 \%$ de l'effectif total d'imagos mâles récoltés. Les espèces secondaires du peuplement imaginal (2 \% $\leq \mathrm{E} \%<10 \%$, LAVILLE, 1971) sont Cladotanytarsus atridorsum, $C$. iucundus, C. pallidus, Tanytarsus debilis, T. usmaënsis, Pseudochironomus prasinatus et Pentapedilum sordens. Toutes les autres espèces (44 espèces) présentent une proportion inférieure à $2 \%$ de l'effectif et peuvent être qualifiées d'espèces rares. Ce peuplement est caractérisé par une diversité relativement faible (indice de diversité de Shannon $\mathrm{H}=3,53$ ).

Le peuplement issu de la récolte des exuvies nymphales apparaît plus diversifié $(H=4,66)$. Une seule espèce, $C$. iucundus, peut être qualifiée d'espèce dominante (figure 2). Le groupe des espèces secondaires est essentiellement constitué par Chironomus anthracinus, Cladotanytarsus atridorsum, Tanytarsus niger, Microtendipes chloris, Psilotanypus rufovittatus et de nombreuses autres espèces représentant chacune entre $2 \%$ et $5 \%$ de l'effectif total (figure 2). Cinquante espèces de ce peuplement présentent un effectif relatif de moins de $2 \%$ de l'effectif total.

Les espèces obtenues en élevage avec les effectifs les plus importants sont $P$. rufovittatus et Einfeldia dissidens (figure 2). Les Tanytarsini sont ensuite bien représentés grâce aux nombreuses émergences de $C$. atridorsum, $C$. nigrovittatus, T. debilis, T. gregarius, T. holochlorus et T. niger. Quelques Chironomini et Tanypodinae complètent la liste des espèces secondaires. La très nette dominance de $P$. rufovittatus conduit à un indice de diversité inférieur à celui obtenu pour le peuplement nymphal $(H=4,31)$.

Parmi les 26 genres répertoriés dans les prélèvements larvaires, quatre taxons dominent à la fois le peuplement endopélique et celui récolté sur les substrats artificiels (figure 3). Ces genres sont Chironomus, Cladotanytarsus, Tanytarsus et Procladius (incluant le sous genre Psilotanypus). En revanche, le genre Einfeldia qui représente le taxon majoritaire du peuplement échantillonné par substrats artificiels est un taxon rare du peuplement endopélique $(E \%=1,5)$. II apparaît également que de nombreux taxons (Glyptotendipes, Microtendipes, 


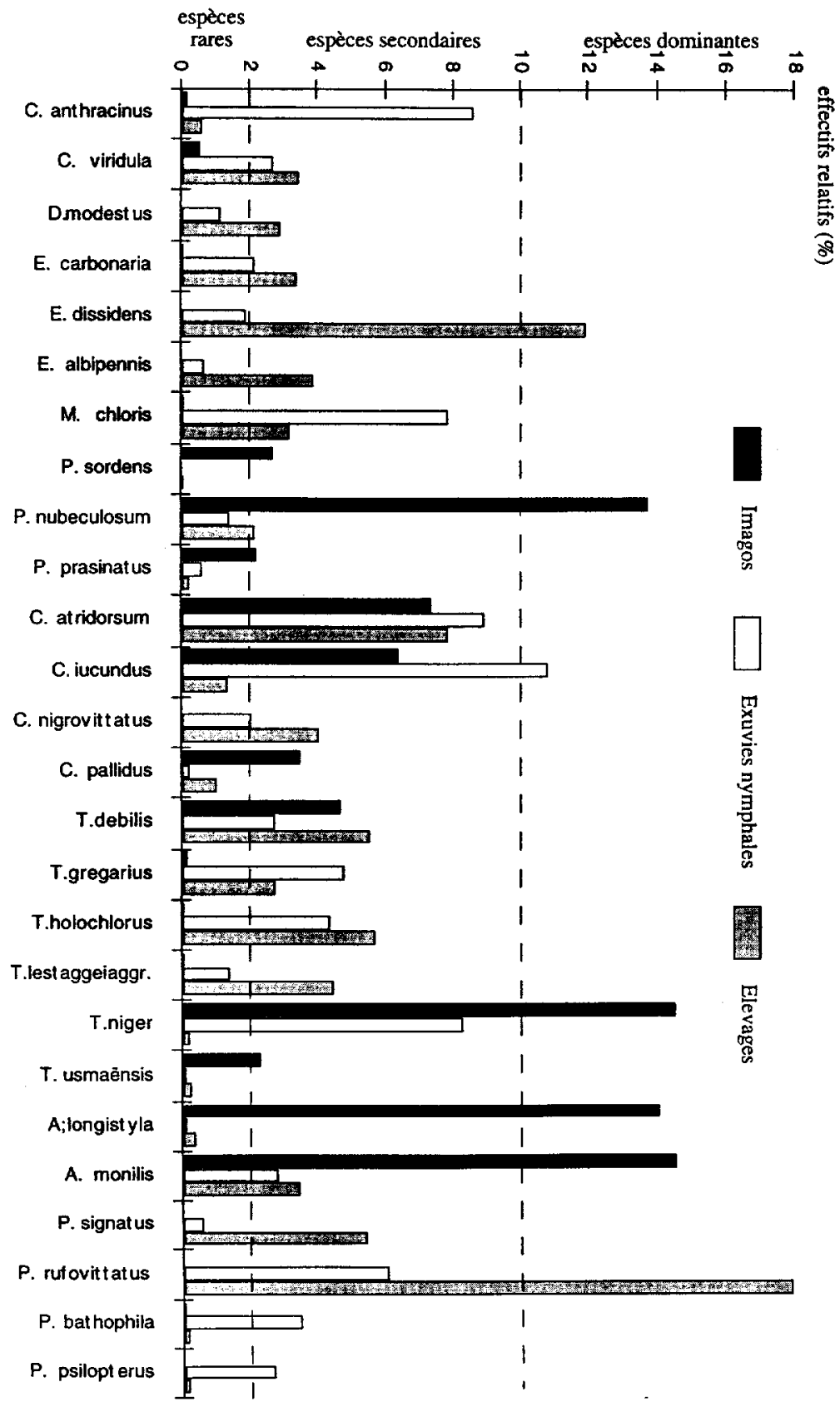

Figure 2 Effectifs relatifs ( $\mathrm{E} \%$ ), dans trois types de peuplements chironomidiens, des espèces principales (dominantes et secondaires) répertoriées dans le lac de l'Abbaye ( $\mathrm{E} \%=2$ dans l'un au moins des peuplements).

Relative number of individuals ( $E \%$ ) of the major contributing chironomid species in three different chironomid communities $(E \%=2$ in at least one of the three communities). 


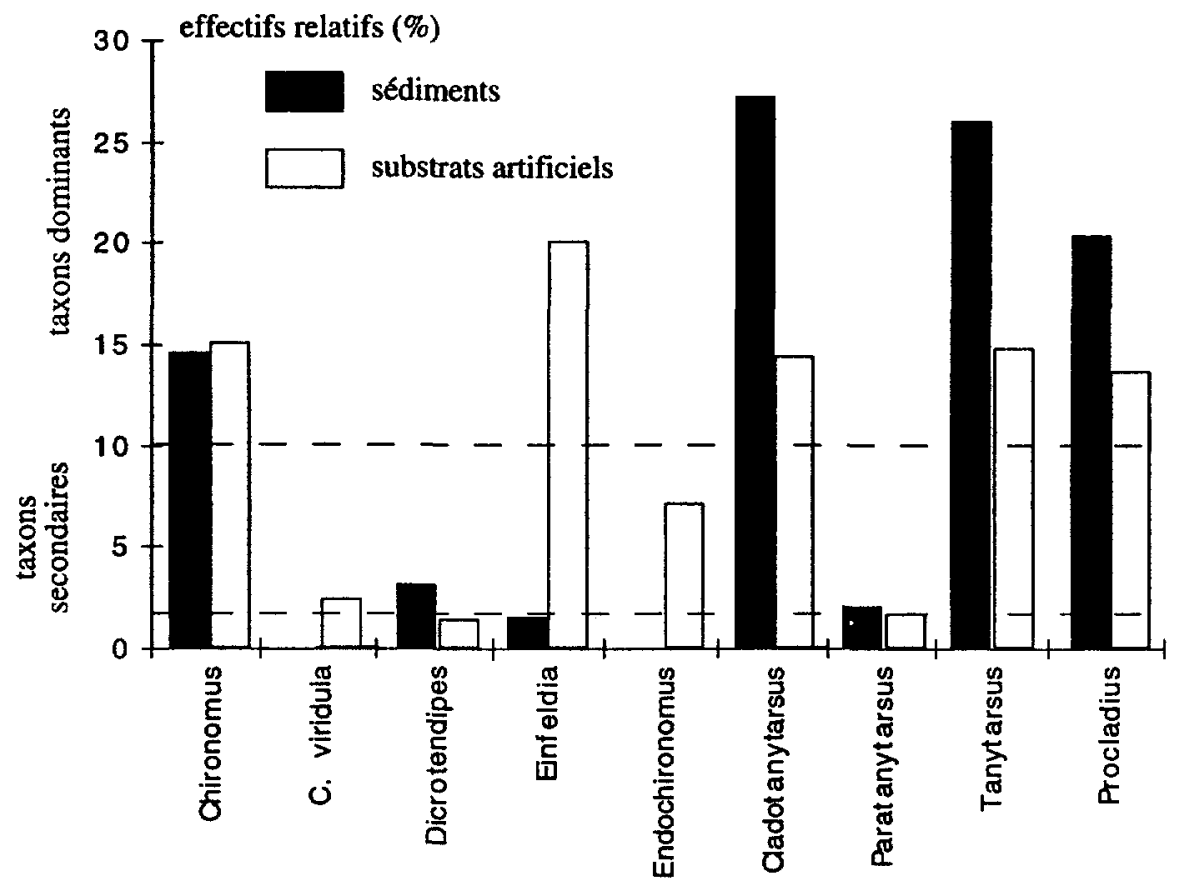

Figure 3. Effectifs relatifs ( $E \%$ ), dans deux types de peuplements larvaires, des principaux genres chironomidiens répertoriés dans le lac de l'Abbaye $(E \%=2$ dans l'un au moins des peuplements).

Relative number of individuals ( $E$ \%) of the major contributing chironomid genera in two different larval communities ( $E \%=2$ in at least one of the two communities).

Paratendipes, Polypedilum, Cricotopus, Parakiefferiella) sont électifs, dans le peuplement larvaire, de la méthode d'échantillonnage par substrats artificiels alors que seuls deux genres (Tribelos et Tanypus) ont été récoltés exclusivement, à un stade larvaire, dans les sédiments (tableau 1).

\section{4 - DISCUSSION}

\subsection{Le peuplement chironomidien global}

Au terme de ce travail, il apparaît que le répertoire spécifique obtenu est caractéristique d'une région montagneuse puisque $82 \%$ des espèces qui le composent sont répertoriées en France par SERRA-TOSIO et LAVILLE (1991) à des altitudes supérieures à $500 \mathrm{~m}$ contre seulement $58 \%$ inventoriées en plaine. Une des particularités de la faune chironomidienne du lac de l'Abbaye est la présence de deux espèces psychrosténothermes (Tanytarsus miriforceps et T. niger) considérées par REISS et FITTKAU (1971) et REISS (1984) comme des espèces reliques 
des périodes de glaciations. Elles constituent des témoins de la formation glaciaire du lac de l'Abbaye.

Avec une variété de 69 espèces, le lac de l'Abbaye semble moyennement diversifié comparé à d'autres écosystèmes tels le lac de Constance (REISS, 1968) ou le lac Innaren (BRUNDIN, 1949) dans lesquels ont été respectivement répertoriées 168 et 140 espèces. Ces deux lacs, présentant un nombre particulièrement élevé d'espèces, sont également des écosystèmes de grande superficie (respectivement 540 et $16 \mathrm{~km}^{2}$ ). L'importance probable de la taille des systèmes sur la variété faunistique n’a pas été démontrée mais certains auteurs ont souligné l'existence dans les grands lacs d'une juxtaposition de plusieurs sous-écosystèmes présentant chacun des caractéristiques habitationnelles différentes (JUGET, 1958 ; DÉVAl et MOLDOVAN, 1983 ; SÄRKKÄ, 1983). Cependant, une comparaison de la variété spécifique chironomidienne du lac de l'Abbaye avec celle d'autres lacs de dimension similaire comme le lac de Petichet (SERRA-TOSIO et GAY, 1978) montre bien que la taille des systèmes ne constitue pas à elle seule le facteur déterminant. En effet, le lac de Petichet, fortement pollué par des apports en nutriments (GAY, 1976) présentait une variété chironomidienne de seulement 18 espèces. En revanche dans le lac de Port-Biehl, plus petit que le lac de l'Abbaye (surface : $16 \mathrm{ha}$ ) mais exempt d'apports anthropiques, le peuplement chironomidien était constitué de 57 espèces (LAVILLE, 1971). Ces observations nous incitent à émettre l'hypothèse que tout système lacustre serait doté d'un potentiel d'espèces, dont l'importance dépend de sa mosaïque d'habitats en relation avec le développement de la zone littorale, susceptible d'être modifié par les conditions physiques et chimiques du milieu.

\subsection{Les méthodes d'échantillonnage}

L'obtention du répertoire spécifique du lac de l'Abbaye par différentes méthodes d'échantillonnage (récolte d'imagos, d'exuvies nymphales et mise en élevage de larves) souligne l'intérêt particulier que présente l'utilisation des exuvies nymphales. La récolte hebdomadaire de ces dernières a permis le recensement de 66 espèces sur les 69 espèces inventoriées. Pour un effort d'échantillonnage similaire, la récolte des imagos est beaucoup moins efficace (seulement 55 espèces pour un effectif considérable). Les avantages pratiques de la collecte des exuvies (diminution des effectifs, du temps de montage et de détermination), par ailleurs fréquemment soulignés (COFFMAN, 1973 ; WILSON et BRIGHT, 1973; WILSON et McGILL, 1977 ; WILSON et WILSON, 1984) expliquent la quasi généralisation de leur utilisation dans les suivis de la qualité des milieux lotiques basés sur la composition des peuplements chironomidiens (McGiLL et al., 1979 ; LAVILLE, 1979 ; WILSON, 1980; LAVILLE et VIAUD-CHAUVET, 1985 ; HAYES et MURRAY, 1988 ; BAZERQUE et al., 1989). De manière assez surprenante, très peu de publications concernant la faune chironomidienne lacustre se réfèrent à l'examen des exuvies nymphales (HUMPHRIES, 1938 ; BUSKENS, 1989).

L'établissement d'un répertoire spécifique par la récolte des imagos au filet entomologique présente deux inconvénients majeurs. Comme le montre la composition particulière du peuplement imaginal obtenu sur le lac de l'Abbaye, comparée aux peuplements obtenus par les exuvies nymphales et les élevages, il semble que cette méthode présente une certaine sélectivité qui entraîne un souséchantillonnage des essaims de petite taille ou les moins accessibles. Ce peuplement imaginal se caractérise en effet par une très faible représentation de 
l'espèce C. anthracinus dont les essaims ont par ailleurs été fréquemment observés à une hauteur de plus de $10 \mathrm{~m}$ et par une proportion très faible des Orthocladiinae $(E \%=0,86)$, formant des essaims peu visibles, comparée à celle obtenue dans le peuplement exuvial $(E \%=10,91)$. De plus, la très forte proportion des espèces $A$ blabesmyia longistyla et $A$. monilis n'est retrouvée dans aucun des autres peuplements échantillonnés. Cette discordance peut s'expliquer par une double origine des imagos, l'une lacustre de faible importance si l'on se réfère au peuplement obtenu par les exuvies nymphales, l'autre issue des systèmes annexes (afférences, marais environnants) et dont les effectifs sont les plus importants. Un phénomène identique est observé pour le genre Polypedilum. Ces résultats mettent en évidence le deuxième inconvénient de la méthode que nous avons par ailleurs récemment démontré (VERNEAUX et ALEYA, 1997 c) et qui consiste en un échantillonnage d'individus ne provenant pas du système étudié. Dans le répertoire spécifique du lac de l'Abbaye (tableau 1) seules ont été conservées les espèces imaginales retrouvées également dans au moins un des autres peuplements échantillonnés. Une étude de la variété spécifique d'un système par la seule récolte des imagos au filet entomologique présente un risque important de surévaluation de la richesse spécifique. Ces inconvénients inhérant à l'étude des imagos peuvent être évités par la mise en place de cages à émergences immergées ou flottantes. Bien que certains auteurs aient utilisé ce matériel d'échantillonnage avec succès, d'autres en revanche ont souligné un certain nombre de difficultés d'utilisation (encombrement du lac, nécessité d'un relevage journalier, effectifs très faibles) nous conduisant à négliger cette méthode d'échantillonnage (DAVIES, 1984).

La mise en élevage des stades pré-imaginaux n'a permis l'obtention que de 48 espèces. Cependant toutes les espèces dominantes ont émergé en élevage et les espèces non obtenues représentent toutes moins de $1 \%$ et même moins de $0,1 \%$ des effectifs du peuplement nymphal. Le système de mise en élevage utilisé, particulièrement simple et ne comprenant aucun contrôle des paramètres mésologiques tels la température, la luminosité ou la photopériode permet d'obtenir le répertoire des espèces principales d'un système lacustre. Cette méthode offre par ailleurs l'avantage, par rapport à la récolte des exuvies nymphales en zone d'accumulation, d'indiquer l'origine bathymétrique des différentes espèces (VERNEAUX et ALEYA, 1997a).

La composition du peuplement larvaire du lac de l'Abbaye montre une nette dominance, quelle que soit la méthode d'échantillonnage utilisée, des genres Chironomus, Cladotanytarsus, Tanytarsus et Procladius (tableau 1). Cependant, la mise en place de substrats artificiels provoque l'apparition de nombreux genres non retrouvés dans le peuplement endopélique et sans doute inféodés à des habitats non sédimentaires. On note également l'absence de quelques genres typiquement endopéliques ainsi que des modifications de la proportionnalité de certains taxons. Ainsi, les substrats artificiels utilisés présentent une forte attractivité pour le genre Einfeldia, ce qui semble en contradiction avec sa qualification de genre inféodé aux sédiments meubles littoraux (WIEDERHOLM, 1983). Les différences observées entre les peuplements benthiques prélevés dans les habitats naturels et ceux échantillonnés par substrats artificiels ont été rapportées depuis longtemps et ont même conduit certains auteurs à remettre en cause la légitimité d'une telle méthode d'échantillonnage (ROSENBERG et RESH, 1982). II apparaît cependant que l'utilisation des substrats artificiels soit appropriée pour l'obtention d'un répertoire chironomidien larvaire plus varié et surtout qu'elle soit adaptée à 
la définition d'un peuplement potentiel essentiellement déterminé, à identité d'habitat, par la qualité de l'eau.

Cette étude pose le problème des méthodes d'échantillonnage utilisées pour la caractérisation des systèmes basée sur les espèces dominantes. En effet, la proportion des différentes espèces récoltées est très variable entre les différents peuplements (figure 2) et les espèces dominantes d'un peuplement constituent les espèces secondaires ou même rares des autres peuplements échantillonnés. Cependant, la confrontation des résultats spécifiques et larvaires permet de conclure que le lac de l'Abbaye est dominé par deux groupes de taxons : les genres Tanytarsus et Cladotanytarsus (figure 3) essentiellement représentés par les espèces $T$. niger, $C$. iucundus et $C$. atridorsum (figure 2) et les genres Chironomus et Procladius (figure 3) parmi lesquels dominent les espèces $C$. anthracinus et $P$. Psilotanypus rufovittatus (figure 2).

\subsection{Caractérisation du lac de l'Abbaye}

La particularité du lac de l'Abbaye réside dans la codominance des deux groupes d'espèces précités dont les significations écologiques diffèrent. Le premier ( $T$. niger, $C$. iucundus et $C$. atridorsum) est constitué d'espèce considérées comme caractéristiques des systèmes à tendance plutôt oligotrophe (BRUNDIN, 1949). Le second, regroupant les espèces $C$. anthracinus et $P$. Psilotanypus rufovittatus conduit à caractériser le lac de l'Abbaye de lac polyhumique désoxygéné (BRUNDIN, 1949) ou encore de lac eutrophe (SAETHER, 1979). Dans le lac de PortBiehl, une association similaire d'espèces (Tanytarsus bathophilus et Chironomus plumosus) a été rapportée (LAVILLE, 1971) et l'auteur a proposé l'hypothèse selon laquelle une telle codominance traduisait une discordance entre le niveau trophique du système et la teneur en matière organique des sédiments. Le lac de l'Abbaye, tout comme le lac de Port-Biehl serait un lac oligotrophe, présentant une faible biomasse phytoplanctonique (Chl a moy. $=3,21 \mu \mathrm{g} \cdot \mathrm{L}^{-1}$; Chl a max $\left.=7,44 \mu \mathrm{g} \cdot \mathrm{L}^{-1}\right)$, mais dont les sédiments accumulent une matière organique d'origine allochtone (lac péritrophe, VERNEAUX et al., 1991), dont la minéralisation est réduite en raison des faibles températures. Ces observations corroborent les conclusions d'autres auteurs qui, à partir de l'examen des communautés d'Oligochètes (LAFONT, 1989 ; LAFONT et al., 1991) et de Mollusques (MOUTHON, 1992) ont respectivement qualifié le lac de l'Abbaye de lac "à faible potentiel métabolique " et de lac « présentant une forte tendance à la dystrophie ». II est également probable que les températures assez peu élevées limitent, dans le temps et dans l'espace, l'impact de la désoxygénation sur la faune chironomidienne.

Au terme de cette étude, il apparaît que l'utilisation des exuvies nymphales constitue un outil particulièrement intéressant pour la caractérisation des systèmes lacustres à partir des espèces chironomidiennes. Afin de préciser les relations existant entre ces espèces et les caractéristiques mésologiques des lacs, nos recherches s'orientent vers une étude de la distribution bathymétrique des espèces chironomidiennes. Pour cela, un échantillonnage des exuvies nymphales à l'aplomb de différentes isobathes couplé à l'analyse de la composition des sédiments et à l'étude de l'oxygénation des interfaces eau-sédiment à ces mêmes profondeurs devra être réalisé. Les conséquences sur l'obtention d'une structure bathymétrique des espèces des phénomènes de dérive nymphale (exuvies ou nymphes) sous l'action des vents ou des courants lacustres, semblent être évitées par la prise en compte d'un seuil d'occurrence dont la valeur dépend de l'occurrence moyenne des espèces sur chaque isobathe (VERNEAUX, 1996). 


\section{REMERCIEMENTS}

Nous remercions vivement le Professeur J. VERNEAUX qui nous a permis de réaliser cette étude et qui, par ses judicieuses remarques a contribué à l'amélioration de ce manuscrit.

\section{RÉFÉRENCES BIBLIOGRAPHIQUES}

AAGAARD K. (1978). The Chironomidae of the exposed zone of Ovre Heimdalsvatn. Holarctic Ecology, 1, 261-265.

BAZZANTI M., SEMINARA M., BALDONI $S$. (1997). Chironomids (Diptera : Chironomidae) fron three temporary ponds of different wet phase duration in Central italy. J. Freshwat. Ecol., 12(1), 89-99.

BAZERQUE M.F., LAVILLE H., BROUQUET Y. (1989). Biological quality assessment in two rivers of the northern plain of France (Picardie) with special reference to chironomid and diatom indices. Acta Biol. Debr. Oecol. Hung., 3, 29-39.

BONOMI G., RUGGIU D. (1966). II Macrobenton profondo del lago di Mergozzo. Mem. Ist. Ital. Idrobiol., 20, 153-200.

BRUNDIN L. (1949). Chironomiden and andere Bodentiere der Südschwedischen Urgebirgsseen. Rep. Inst. Freshwat. Res. Drottnigholm, 30, 1-914.

BRINKHURST O.A. (1974). The benthos of Lakes. McMillan Press. Ltd London and Basingstoke : $190 \mathrm{p}$.

BUSKENS R.F.M. (1989). Monitoring chironomid larvae and exuviae in the Beuven, a soft water pool in the Netherlands, and comparisons with paleolimnological data. Acta Biol. Debr. Oecol. Hung., 3, 41-50.

CASAS J.J., VILCHEZ-QUERO A. (1996). Chironomid assemblages of three endoreic karstic lagoons (Southern Spain) determined by collection of pupal exuviae : Importance of the water mineralization and sediment characteristics. Int. Rev. ges. Hydrobiol., 81(4), 555-564.

CASEY R.J., KENDALL A.S. (1996). Comparisons among colonization of artificial substratum types and natural substratum by benthic macroinvertebrates. Hydrobiofogia, 341, 57-64.

COFFMAN W.P. (1973). Energy flow in a woodland stream ecosystem. The taxonomic composition and phenology of the Chironomidae as determined by the collection of pupal exuviae. Arch. Hydrobiol., 71, 281-322.

Cranston P.S. (1995). The Chironomidae. The biology and ecology of non-biting midges. Armitage P., Cranston P.S. and Pinder L.C.V. Ed : $572 \mathrm{p}$.

DAVIES I.J. (1984). Sampling aquatic insect emergence. In : A manual on methods for the Assessment of secondary productivity in fresh waters. Dowing A.J., Rigler F.H. (ed). Blackwell scientific publications. Oxford, London, Edinburgh, Boston, Melbourne: 161-221.

DÉVAI G., MOLDOVAN J. (1983). An attempt to trace eutrophication in a shallow lake (Balaton, Hungary) using Chironomids. Hydrobiologia, 103, 169-175.

DINSMORE W., PREPAS E.E. (1997). Impact of hypolimnetic oxygenation on profundal macroinvertabrates in an eutrophic lake in Central Alberta. I. Change in macroinvertabrate abundance and diversity. Can. J. Fish. Aquat. Sci., 54, 2157-2169.

EVRARD M. (1994). Évolution journalière de la dérive des exuvies nymphales de Chironomidae (Diptera) dans une rivière salmonicole (Le Sauron, Belgique). Belg. J. Zool., 124(2), 115-126.

FEND S. V., CARTER J. L. (1995). The relationship of habitat characteristics to the distribution of Chironomidae (Diptera) as measured by pupal exuviae collections in a large river system. J. Freshwat. Ecol., 10(4), 343-359. 
FENCHEL T. (1987). Ecology of protozoa. Science Tech. Publishers, Madison, Wisconsin/Springer - Verlag, New York : 197 p.

FRANQUET E., PONT D. (1996). Pupal exuviae as descriptors of the chironomid (Diptera: Nematocera) communities of large rivers. Arch. Hydrobiol., 138(1), 77-98.

GAY C. (1976). Contribution à l'étude écologique et à l'aménagement d'un lac de montagne: le lac de Petichet (Isère) (altitude: $923 \mathrm{~m}$ ). Thèse de Doctorat de $3^{e}$ cycle, Univ. Grenoble : 124 p.

GIMARET H. (1995). L'utilisation de substrats artificiels pour l'analyse biologique comparée des lacs. Durée d'exposition. Hydroé col. Appl., 7(1-2), 1-18.

GRIMAS U. (1961). The bottom fauna of natural and impounded lakes in northen Sweden (Ankanvattnet and Blasjön). Rep. Inst. Freshwat. Res. Drottnigholm, 42, 183-237.

HAYES B.P., MURRAY D.A. (1988). Diel variation in chironomid emergence and implication for the use of pupal exuviae in river classification. Verh. Internat. Verein. Limnol., 23, 1-6.

HIRABAYASHI K., HAYASHI H. (1996). Seasonnal variation of Chironomus nipponensis (Diptera) voltinism in the deep mesotrophic Lake Kizaki, Japon. Arch. Hydrobiol., 138(2), 229-244.

HUMPHRIES C.F. (1938). The chironomid fauna of the Grosser Plöner See, the relative density of its members and their emergence period. Arch. Hydrobiol., 33, 535-584.

HYNES H.B.N. (1970). The Ecology of Running Waters. Liverpool Univ. Press : 555 p.

JOHNSON K.R., WIEDERHOLM T. (1989). Classification and ordination of profundal macroinvertabrate communities in nutrient poor, oligo-mesohumic lakes in relation to environmental data. Freshwat. Biol., 21, 375-386.

JOHNSON K.R., WIEDERHOLM T., ERIKSSON L. (1993). Classification of littoral macroinvertabrate communities of Swedish reference lakes. Verh. Internat. Verein. Limnol., 25, 512-517.

JUGET J. (1958). Recherches sur la faune de fond dun Léman et du lac d'Annecy. Ann. stat. Cent. Hydrobiol appl., 7, 9-94.

KRISTENSEN E. (1988). Benthic fauna and biogeochemical processes in marine sediments : microbial activities and fluxes. In T.H. Blackburn \& Sörensen (eds), Nitrogen cycling in coastal marine environments. Wiley \& Sons, Chichester.

LAFONT M. (1989). Contribution à la gestion des eaux continentales : utilisation des Oligochètes comme descripteurs de l'état biologique et du degré de pollution des eaux et des sédiments. Thèse de Doctorat ès Sciences, UCBL Lyon $1: 311$ p. + annexes $92 \mathrm{p}$.

LAFONT M., JUGET J. \& ROFES G. (1991). Un indice biologique lacustre basé sur l'examen des Oligochètes. Rev. Sci. Eau, 4, 253-268.

LANGTON P.H. (1991). A key to pupal exuviae of West Palaearctic Chironomidae. Privately published, 386 p.

LAVILLE H. (1971). Recherches sur les Chironomides (Diptera) lacustres du massif de Néouvielle (Hautes-Pyrénées). Première partie : Systématique, Écologie, Phénologie. Ann. Limnol., 7, 173-332.

LAVILLLE H. (1974). Utilisation des substrats artificiels pour l'étude de la faune macrobenthique de la zone littorale rocheuse des lacs de montagne. Ann. Limnol., 10(2), 163-171.

LAVILLE H. (1979). Étude de la dérive des exuvies nymphales de chironomides au niveau du confluent Lot-Truyère. Ann. Limnol., 15(2), 155-180.

LAVILLE H., VIAUD-CHAUVET M. (1985). Étude comparée de la structure des peuplements de chironomidés dans cinq rivières du Massif Central : relation entre cette structure et la qualité des eaux. Verh. Internat. Verein. Limnol., 22, 2261-2269.

LINDEBERG B. (1964). The swarm of males as a unit for taxonomic recognition in the chironomids (Diptera). Ann. Zool. Fenn., 1, 72-76.

LINDEGAARD C. (1979). Bathymetric distribution of Chironomidae (Diptera) in the oligotrophic Lake Thingvallavatn, Iceland. Chironomidae. Ecology, Systematics, Cytology and Physiology. Ed. D.A. Murray : 225-232.

MACAN T.T. (1961). A review of running water studies. Verh. internat. Verein. Limnol., 14, 587-602.

MACAN T.T. (1970). Biological studies of the English lakes. Longman Group Ltd London : $260 \mathrm{p}$. 
MALÉSIEUX G. (1976). Utilisation des substrats artificiels benthiques pour la détermination de la qualité des eaux. Mem. DESS, Univ. Franche-Comté, 55 p.

MCGILL J.D., WILSON R.S., BRAKE A.M. (1979). The use of chironomid pupal exuviae in the surveillance of sewage pollution within a drainage system. Wat. Res., 13, 887-894.

MOUTHON J. (1992). Peuplements malacologiques lacustres en relation avec la physico-chimie de l'eau et des sédiments. Ann's Limnol., 28, 109-119.

OLIVER D.R. (1960). The macroscopic bottom fauna of Lac la Ronge, Saskatchewan. J. Fish. Res. Bd Can., 17, 607 624.

PINDER D. R. (1978). A key to the adult males of British Chironomidae. Fresh. Wat. Biol. Assoc., Scient. Publ., 37, $169 \mathrm{p}$.

PORTER K.G., Sherr E.B., Sherr B.F., Pace M., Sauders R.W. (1985). Protozoa in planktonic food webs. J. Protozool., 32, 409-415.

REISS F. (1968). Verbreitung lakustrischer Chironomiden (Diptera) des Alpengebietes. Ann. Zool. Fenn., 5, 119-125.

REISS F. (1984). Die Chironomidenfauna (Diptera, Insecta) des Osterseegebietes in Oberbayern. Ber. Ann., 8, 186-194.

REISS F., Fittkau E.J. (1971). Taxonomie und Ökologie europäisch Verbreiteter Tanytarsus Arten (Chironomidae, Diptera). Arch. Hydrobiol. Suppl., 40(1-2), 75-200.

RIERADEVALL M., PRAT N. (1991). Benthic fauna of Banyoles Lake (NE Spain). Vehr. internat. Ver. Limnol., 24, 1020-1023.

AOSENBERG D.M., RESH V.H. (1982). The use of artificial substrates in the study of freshwater benthic macroinvertebrates. In Artificial Substrates. J. Cairns J.V. (ed.). Ann. Arbor. Science, 175-235.

RUSE L.P. (1995). Chironomid community structure deduced from larvae and pupal exuviae of a chalk stream. Hydrobiologia, $315,135-142$.

SAETHER O. A. (1979). Chironomid communities as water quality indicators. Holarct. Ecol., 2, 65-74.

SÄRKKÄ J. (1983). A quantitative ecological investigation of the littoral zoobenthos of an oligotrophic Finnish lake. Ann. Zool. Fenn., 20, 157-178.
SERRA-TOSIO B. (1971). Contribution à l'étude taxonomique, phylogénétique, biogéographique et écologique des Diamesini (Diptera, Chironomidae) d'Europe. Mem. Thesis, Univ. Grenoble. Tome I : 303 p., Tome II : 462 p.

SERRA-TOSIO B., GAY C. (1978). Les Diptères Chironomidés et Chaoboridés du lac de Petichet (lsère). Trav. Lab. Hydrobiol., 69-70, 97-105.

SERRA-TOSIO B., LAVILLE H. (1991). Liste annotée des Diptères Chironomidés de France continentale et de Corse. Ann. Limnol., 27(1), 37-74.

SHERK T., RAU G. (1996). Emergence of Chironomidae from Findley Lake in the conjferous forest of the Cascade Mountains after early and late thaws. Hydrobiologia, 318, 85-101.

SHERR B.F., SHERR E.B. (1984). Role of heterotrophic protozoa in carbon and energy flow in aquatic ecosystems. In M.J. Klugg \& C.A. Reddy (eds.), Currents perspectives in Microbial Ecology. Amer. Soc. Microbiol. Washington, 412-423.

SHERR E.B., SHERR B.F., PAFFENHOFER G.A. (1986). Phagotrophic protozoa as food for metazoans: a "missing" trophic brick in marine pelagic food webs ? Mar. Microbiol. Food Webs, 1, 61-80.

SLACK H.D. (1965). The profundal fauna of Loch Lomond, Scotland. Proc. R. Soc. Edinburgh, 27, 55-76.

VEIJOLA H., MERILÄINEN J.J., MARTTILA V. (1996). Sample size in the monitoring of benthic macrofauna in the profundal of lakes: evaluation of the precision of estimates. Hydrobiologia, 322, 301-315.

VERNEAUX J., REMY F., VIDONNE A., GUYARD A. (1987). Caractères généraux des sédiments de 10 lacs jurassiens. Rev. Sci. Eau, 6, 107-128.

VERNEAUX J., VIDONNE A., REMY F., GUYARD A. (1991). Particules organiques et rapport $\mathrm{C} / \mathrm{N}$ des sédiments des lacs du Jura. Ann. Limnol., 27, 175-190.

VERNEAUX J., VERNEAUX V., GUYARD A. (1993). Classification biologique des lacs jurassiens à l'aide d'une nouvelle méthode d'analyse des peuplements benthiques. I. Variété et densité de la faune. Ann. Limnol., 29(1), 59-77.

VERNEAUX V. (1996). Structure, dynamique spatiale et temporelle du peuplement chironomidien du lac de l'Abbaye (massif du 
Jura) et approche typologique. Relation entre la topologie larvaire et la structuration bathymétrique des peuplements émergents. Mém. Thèse $3^{e}$ cycle, Univ. Franche-Comté : 187 p. + annexes.

VERNEAUX V., ALEYA L. (1997a). Spatial and temporal distribution of chironomid larvae (Diptera : Nematocera) at the sediment-water interface in Lake Abbaye (Jura, France). Hydrobiologia (in press).

VERNEAUX V., ALEYA L. (1997b). Bathymetric distributions of chironomid communities in ten French lakes : Implication on lake classification. Arch. Hydrobiol. (in press).

VERNEAUX V., ALEYA L. (1997c). Diptères chironomidés et caractérisation des lacs. Ann. Biol., 25(4), 220-235.

WIEDERHOLM T. (1983). Chironomidae of the Holarctic region. Keys and diagnoses. Part 1. Larvae. Ent. Scand. Suppl., 18, 1-457.
WIEDERHOLM T., ERIKSSON L. (1977). Benthos of an acid lake. Oikos, 29, 261267.

WILSON R.S. (1980). Classitying rivers using chironomid pupal exuviae. In Chironomidae. Ecology, Systematic, Cytology and Physiology. Murray D.A. (Ed), Pergamon Press, Oxford, New York : $354 \mathrm{p}$.

WILSON R. S., BRIGHT P. L. (1973). The use of pupal exuviae for characterizing streams. Freshwat. Biol., 3, 283-302.

WILSON R.S., MCGILL J.D. (1977). Chironomid pupal exuviae in the River Chew. Freshwat. Biol., 7, 9-17.

WILSON R. S., WILSON S. E. (1984). A survey of the distribution of Chironomidae (Diptera, Insecta) of the River Thine by sampling pupal exuviae. Hydrobiol. Bull., 18, 119-132. 